\title{
Surfing Multiple Conformation-Property Landscapes via Machine Learning: Designing Single-Ion Magnetic Anisotropy. Supplementary Information.
}

\author{
${ }^{1}$ Alessandro Lungh ${ }^{*}$ and ${ }^{1}$ Stefano Sanvito \\ ${ }^{1}$ School of Physics, AMBER and CRANN Institute, Trinity College, Dublin 2, Ireland
}

\section{ANISOTROPY DECOMPOSITION IN SPHERICAL HARMONICS}

The decomposition of the Cartesian tensor $D_{i j}$ into a 2-rank spherical harmonics $T^{m}$, with $m=-2,2$, is done accordingly to the relations

$$
\begin{aligned}
& T^{0}=\frac{1}{\sqrt{6}}\left(3 D_{33}-\left(D_{11}+D_{22}+D_{33}\right)\right), \\
& T^{-1}=\frac{1}{2}\left(D_{13}+D_{31}-i\left(D_{23}+D_{32}\right)\right), \\
& T^{1}=-\frac{1}{2}\left(D_{13}+D_{31}+i\left(D_{23}+D_{32}\right)\right), \\
& T^{-2}=\frac{1}{2}\left(D_{11}-D_{22}-i\left(D_{12}+D_{21}\right)\right), \\
& T^{2}=\frac{1}{2}\left(D_{11}-D_{22}+i\left(D_{12}+D_{21}\right)\right) .
\end{aligned}
$$

\section{AB INITIO CALCULATIONS}

The ORCA software [1 has been employed for all the calculations. We have used the basis sets def2-TZVP for $\mathrm{C}, \mathrm{N}$ and $\mathrm{S}$ species and the def2-SVP for $\mathrm{C}$ and $\mathrm{H}$ species. The def2-TZVP/C auxiliary basis set has been used for all the elements. The calculations of the $\mathbf{D}$ tensor have been carried out at the CASSCF level of theory, with a $(7,5)$ active space and spin-orbit contributions included through quasi-degenerate perturbation theory. The calculations of the conformational energy has been performed at the DFT level with the PBE functional [2].

\section{SUPERVISED LEARNING.}

The coefficients $\alpha$ of the machine learning model where determined by linear Ridge regression:

$$
\min _{\left\{\alpha_{j}\right\}}\left[\left\|T_{\mathrm{QM}}^{m}\left(\left\{r_{i}\right\}\right)-T_{\mathrm{ML}}^{m}\left(\left\{r_{i}\right\},\left\{\alpha_{j}^{m}\right\}\right)\right\|^{2}+\lambda\left\|\left\{\alpha_{j}^{m}\right\}\right\|^{2}\right] .
$$

where the first term corresponds to the canonical leastsquare-fitting of the $T_{\mathrm{QM}}^{m}$ first principles reference values, and the second one to the regularization term. The optimal value of $\lambda$ was chosen as to minimise the error on the validation set. The code LAMMPS 3 has been used to generate the bi-spectrum components. In all cases the order $2 J=8$ for the bi-spectrum components, corresponding to 56 elements per atomic species, has been used. The number of atomic species is a variable that can be adapted to increase the accuracy of the model and does not necessarily need to correspond to the chemical elements. The regression of $\mathrm{Co}(\mathrm{pdms})_{2}$ 's energy was computed by increased the number of atomic species to nine by discriminating chemically-inequivalent chemical elements. Conversely, the regression of $\mathrm{Co}(\mathrm{pdms})_{2}$ 's magnetic anisotropy was carried out by only considering the atoms within the radial cutoff distance from the Co atom. In the latter case the correspondence between atomic species and chemical elements was used. The radial cutoff $R_{\text {cut }}$ used to build the bi-spectrum components have been optimised as to minimise the overall error on the training/validation set and fixed to $3.5 \AA$ for $\mathrm{Co}(\mathrm{pdms})_{2}$ 's magnetic anisotropy, $3.1 \AA$ for $\mathrm{Co}(\mathrm{pdms})_{2}$ 's energy and $4.5 \AA$ for both $\mathrm{FeCl}_{x}$ 's energy and magnetic anisotropy. The definition of bi-spectrum components gives the possibility to differentiate atomic kinds with weights and atomic radii 4 . In this work we have set all the weights to unity and kept all the atomic radii equal to 0.5 . The latter condition corresponds to using the same $R_{\text {cut }}$ for every species. The covariancy of Ridge regression for tensorial properties requires the estimation of the amount of rotation between each configuration and the reference molecular orientations. This was estimated by applying the Eckart-Sayvets conditions [5]. This approach provide the rotation matrix that brings the Cartesian coordinates of a structure into those of a rotated one by taking into account that a rigid translation and an intra-molecular motion might also have occurred. This rotation matrix can be interpreted in terms of Euler's angles. The latter are then used to compute the Wigner rotation matrix that appears in Eq. 5 of the main text.

\section{PARTICLE SWARM OPTIMIZATION.}

The $\alpha-t h$ particle in the swarm corresponds to a vector $p^{\alpha}$ that stores the position of every atom in space. The vectors $p^{\alpha}$ are propagated by summing them with the particle velocity $v_{\alpha}$. The velocity of each particle

\footnotetext{
*lunghia@tcd.ie
} 
in the swarm was updated at each $i-t h$ step with a simple scheme: $v_{\alpha}^{i+1}=\omega v_{\alpha}^{i}+\Gamma\left[c_{1} p_{\text {best }}^{\alpha}+c_{2} p_{\text {best }}\right] . p_{\text {best }}^{\alpha}$ corresponds to the vector $p^{\alpha}$ that scored the best in the history of the particle $\alpha$, while $p_{\text {best }}$ correspond to the vector that scored the best among all the particle in the swarm. The coefficients $\omega$ and $\Gamma$ were chosen as 0.7 and 1.70 , respectively. The coefficients $c_{1}$ and $c_{2}$ are random number in the range [0:1]. Tests with different values of $\gamma$ and number of particles have been carried out with no significant difference in the results.
[1] Frank Neese. The ORCA program system. Wiley Interdiscip. Rev. Comput. Mol. Sci., 2(1):73-78, jan 2012.

[2] John P Perdew, Kieron Burke, and Yue Wang. Generalized gradient approximation for the exchange-correlation hole of a many-electron system. Phys. Rev. B, 54:533-539, 1996.

[3] Steve Plimpton. Fast Parallel Algorithms for Shor-Range Molecular Dynamics. J. Comput. Phys., 117:1-19, 1995.

[4] Aidan P. Thompson, Laura P. Swiler, Christian R. Trott, Stephen M. Foiles, and Garritt J. Tucker. A Spectral
Analysis Method for Automated Generation of QuantumAccurate Interatomic Potentials. J. Comput. Phys., 285:316-330, 2015.

[5] Natale Neto and Luca Bellucci. A new algorithm for rigid body molecular dynamics. Chem. Phys., 328(1-3):259$268,2006$. 